\title{
Hypoxia-Induced Oxidative Stress in Ischemic Retinopathy
}

\author{
Suk-Yee Li, ${ }^{1}$ Zhong Jie Fu, ${ }^{1}$ and Amy C. Y. Lo ${ }^{1,2}$ \\ ${ }^{1}$ Eye Institute, Li Ka Shing Faculty of Medicine, The University of Hong Kong, 21 Sassoon Road, \\ Pokfulam, Hong Kong \\ ${ }^{2}$ Research Centre of Heart, Brain, Hormone and Healthy Aging, Li Ka Shing Faculty of Medicine, \\ The University of Hong Kong, Pokfulam, Hong Kong \\ Correspondence should be addressed to Amy C. Y. Lo, amylo@hkucc.hku.hk
}

Received 2 May 2012; Revised 24 August 2012; Accepted 17 September 2012

Academic Editor: Remi Mounier

Copyright (c) 2012 Suk-Yee Li et al. This is an open access article distributed under the Creative Commons Attribution License, which permits unrestricted use, distribution, and reproduction in any medium, provided the original work is properly cited.

Oxidative stress plays a crucial role in the pathogenesis of retinal ischemia/hypoxia, a complication of ocular diseases such as diabetic retinopathy (DR) and retinopathy of prematurity (ROP). Oxidative stress refers to the imbalance between the production of reactive oxygen species (ROS) and the ability to scavenge these ROS by endogenous antioxidative systems. Free radicals and ROS are implicated in the irreversible damage to cell membrane, DNA, and other cellular structures by oxidizing lipids, proteins, and nucleic acids. Anti-oxidants that can inhibit the oxidative processes can protect retinal cells from ischemic/hypoxic insults. In particular, treatment using anti-oxidants such as vitamin $\mathrm{E}$ and lutein, inhibition of nicotinamide adenine dinucleotide phosphate oxidase (NADPH oxidase) or related signaling pathways, and administration of catalase and superoxide dismutase (SOD) are possible therapeutic regimens for DR, ROP, and other retinal ischemic diseases. The role of oxidative stress in the pathogenesis of DR and ROP as well as the underlying mechanisms involved in the hypoxia/ischemia-induced oxidative damage is discussed. The information provided will be beneficial in understanding the underlying mechanisms involved in the pathogenesis of the diseases as well as in developing effective therapeutic interventions to treat oxidative stress-induced damages.

\section{Introduction}

The retina is highly susceptible to oxidative damage by reactive oxygen species (ROS). Besides having the highest oxygen consumption in the body [1], the retina is also prone to photo-oxidation due to its constant exposure to incoming light. The high oxygen consumption and endless light exposure in the retina may in turn generate ROS. Moreover, the high lipid content in the retina (due to abundant polyunsaturated fatty acids in the photoreceptor outer segment) makes it prone to lipid peroxidation. During pathological conditions such as retinal ischemia, the imbalance between the production of ROS and the ability to scavenge these ROS by endogenous antioxidant systems is exaggerated. ROS triggers several signaling pathways, affects DNA and lipids inside the cell, and subsequently leads to cell death. Antioxidants that can inhibit or prevent the oxidative processes can protect retinal cells from ischemic damage.

\section{Sources of Oxidants}

A free radical is an atom, a molecule, or an ion having unpaired electrons. Due to the presence of the "free" electron in the outer shell, free radical is chemically very unstable and reactive. In order to achieve stability, free radical will participate in further reduction and oxidation reactions. Therefore, production of one free radical leads to further radical formation via sequential chain reactions [2]. Free radicals that are derived from oxygen are ROS, being one of the major contributors of oxidative stress. They include superoxide anion $\left(\mathrm{O}_{2}{ }^{-}\right)$, perhydroxyl radical (also known as hydroperoxyl radical, $\mathrm{HO}_{2}$ ), and hydroxyl radical $(\mathrm{OH})$. Superoxide is formed by either the enzymatic reduction of oxygen by nicotinamide adenine dinucleotide phosphate oxidase (NADPH oxidase) or nonenzymatic reaction of mitochondrial respiration [3]. It is converted into hydrogen peroxide $\left(\mathrm{H}_{2} \mathrm{O}_{2}\right)$ enzymatically by superoxide dismutase (SOD) or 
nonenzymatically to $\mathrm{H}_{2} \mathrm{O}_{2}$ and singlet oxygen [3, 4]. Singlet oxygen is a powerful oxidant and is able to oxidize lipids, proteins and nucleic acids [5]. Perhydroxyl radical is the protonated form of superoxide, acting as an oxidant and reacts with polyunsaturated fatty acids in the membrane lipid bilayer, thereby initiating lipid peroxidation. Hydroxyl radical is another reactive radical which can be formed either from the reaction of singlet oxygen and $\mathrm{H}_{2} \mathrm{O}_{2}$ by the HaberWeiss reaction [6] or from the interaction between $\mathrm{H}_{2} \mathrm{O}_{2}$ and reduced transition metals such as ferrous ions by the Fenton reaction $[3,6,7]$. Generation of superoxide, singlet oxygen and hydroxyl radical causes detrimental effects on the physiological states of cells by cleaving covalent bonding in protein and carbohydrates, leading to lipid peroxidation and compromising the integrity of cell membrane $[3,6,7]$.

Another free radical, nitric oxide (NO) is generated from the oxidation of the guanidine group of L-arginine catalyzed by the enzyme nitric oxide synthase (NOS). NO is in fact a signaling molecule. Moreover, NO can react with superoxide to from a strong oxidant, peroxynitrite (ONOO), one of the reactive nitrogen species (RNS). Similar to ROS, excessive production of RNS (nitrosative stress) results in nitrosylation, causing deleterious effects such as lipid peroxidation, DNA damage and SOD inactivation [8]. Therefore, the toxic effects of ONOO and NO should also be taken into account in developing the antioxidative strategy [9].

\section{Anti-Oxidative Mechanisms and Oxidative Stress}

In physiological condition, ROS and RNS are products of normal cellular metabolism. ROS can be efficiently scavenged by the intrinsic antioxidant defense mechanisms. Manganese superoxide dismutase (MnSOD), copper/zinc superoxide dismutase $(\mathrm{Cu} / \mathrm{Zn} \mathrm{SOD})$, catalase, and glutathione peroxidase $(\mathrm{GPx})$ are members of the enzymatic antioxidants while ascorbic acid (vitamin C), $\alpha$-tocopherol (vitamin $\mathrm{E})$, glutathione $(\mathrm{GSH})$, and $\beta$-carotene are nonenzymatic antioxidants. For example, MnSOD and $\mathrm{Cu} / \mathrm{Zn}$ SOD convert superoxide to $\mathrm{H}_{2} \mathrm{O}_{2}$, which is then efficiently changed to water by GPx or catalase $[10,11]$. On the other hand, vitamin $\mathrm{E}$, vitamin $\mathrm{C}$ and $\beta$-carotene act as radical scavengers. Vitamin E also reduces and removes lipid peroxyl radical, a product of the reaction between lipid radical (a result of electron extraction from polyunsaturated fatty acid by hydroxyl radical) with molecular oxygen and an initiator of lipid peroxidation within the cell membrane. Subsequently, vitamin $\mathrm{E}$ is regenerated by vitamin $\mathrm{C}$. Therefore, both vitamin $\mathrm{E}$ and vitamin $\mathrm{C}$ help to cease the lipid peroxidation chain reactions involving lipid peroxyl radicals [12]. GSH, a major cellular redox buffer, can directly scavenge hydroxyl radical and singlet oxygen. Through the action of GPx, GSH can also remove $\mathrm{H}_{2} \mathrm{O}_{2}$ and lipid peroxides. Moreover, GSH helps to regenerate both vitamin $\mathrm{E}$ and vitamin $\mathrm{C}$ back to their active forms. Normally, a balance is maintained between the levels of oxidants and antioxidants, which is essential for survival.
During ischemic injury, there is overproduction of free radicals that in turn leads to further production of radicals through chain reactions $[10,12]$. The imbalance between the overproduction and scavenge of ROS/RNS leads to oxidative stress/nitrosative stress. The excess ROS can damage cellular lipids, proteins, or DNA and in turn inhibit their normal function. ROS also disturbs normal functioning of mitochondria by opening the mitochondrial membrane permeability transition pores (PTPs), leading to energy uncoupling and further ROS production [13]. This also induces the release of cytochrome $c$, leading to apoptosis $[10,14,15]$. In addition, superoxide reacts with $\mathrm{NO}$ to form ONOO anion and in turn causes oxidation, lipid peroxidation, and subsequently cellular damage [8]. Accumulation of ONOO and free radicals in the nucleus also leads to DNA damage [9]. Indeed, an increased level of nitrotyrosine, the nitration product of $\mathrm{ONOO}$, is observed after retinal ische$\mathrm{mia} /$ reperfusion (I/R) injury and is attributed to cell death $[16,17]$.

Retinal ischemia is a common feature in ischemic retinopathy such as diabetic retinopathy (DR) and retinopathy of prematurity (ROP). In both ocular diseases, there is initially an impairment in the normal retinal blood supply while the subsequent formation of abnormal new blood vessels (retinal neovascularization) further worsens the condition. The presence of ischemia and consequently oxidative stress makes the already vulnerable retina more prone to oxidative damage.

\section{Oxidative Stress and the Pathogenesis of Diabetic Retinopathy}

DR is one of the major causes of blindness worldwide. Most importantly, all patients with diabetes mellitus are at risk. The longer the patient has diabetes, the higher the chance of developing DR. As the total number of people with diabetes, estimated to be 171 million worldwide in 2000 [18] is rising, DR causes enormous economic and social burden to the society. In particular, DR affects working-age adults. This poses extra need and effort in treating the disease. Despite extensive research, the promising therapeutics has yet to come. Kowluru and Zhong have recently published an excellent review in antioxidant therapy for age-related macular degeneration (AMD) and DR, providing evidence on the positive potential of antioxidant therapy clinical trials in DR treatment [18] (Figure 1).

Basement membrane thickening, pericytes loss, microaneurysms, blood-retinal barrier breakdown, and neovascularization are pathological features of DR [19]. Microvascular complication is a manifestation of DR which leads to retinal ischemia/hypoxia and exacerbates the condition $[20,21]$. Four major mechanisms are involved in development of pathologies in DR: increased polyol pathway flux, increased formation of advanced glycation end-products (AGEs), activation of protein kinase $\mathrm{C}$ (PKC) isoforms, and increased hexosamine pathway flux [22].

Increasing body of evidence shows that these metabolic mechanisms are associated with excess production of ROS and depletion of antioxidants in DR [19]. Firstly, aldose 


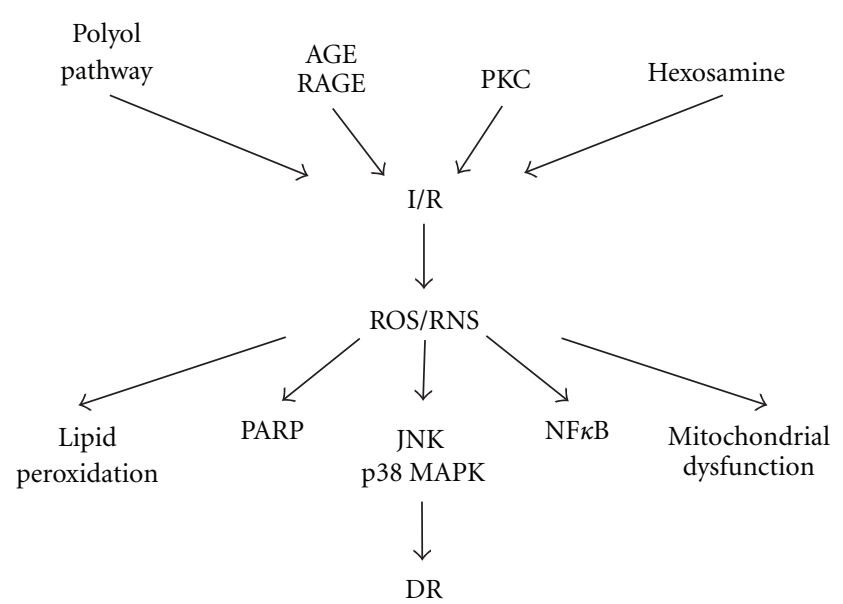

FIGURE 1: Schematic showing the relationship between oxidative stress and DR. The four major mechanisms involved in DR: increased polyol pathway flux, increased AGE formation, activation of PKC pathway, and increased hexosamine pathway flux results in $\mathrm{I} / \mathrm{R}$ and increased production of ROS/RNS, thereby changing the levels of JNK/p38 MAPK, PARP, and NF $\kappa B$, increasing lipid peroxidation as well as leading to mitochondrial dysfunction.

reductase (AR) is the first and rate-limiting enzyme in the polyol pathway. In a hyperglycemic condition, AR reduces glucose to sorbitol, consuming the cofactor NADPH. Sorbitol is then oxidized to fructose by the enzyme sorbitol dehydrogenase. As NADPH is used for generating the intracellular antioxidant GSH, a reduction in the availability of NADPH exacerbates intracellular oxidative stress. The increase in enzymatic activity of AR during hyperglycemia further worsens the situation of DR $[22,23]$. On the other hand, inhibition of AR activity, either by specific inhibitor or gene deletion, may have a therapeutic role in preventing the progression of diabetic retinopathy [24-26] and ischemiarelated ocular diseases $[21,27,28]$. Application of the AR inhibitor, fidarestat, is beneficial in decreasing the concentrations of sorbitol and fructose and accumulation of leukocytes in diabetic retina [24]. Secondly, AGEs and its receptor, RAGE, can also cause oxidative stress. AGEs induce oxidative damage by overproduction of superoxide in neuronal culture through the activation of NADPH oxidase in a $\mathrm{PKC} \delta$-dependent manner [29-31]. Increased intracellular formation of AGE and RAGE activates a cascade of signaling proteins and attributes to intracellular redox imbalance, which leads to overproduction of ROS [2931]. On the other hand, inhibition of AGE formation and suppression of the RAGE-mediated downstream pathway have been suggested to be the feasible therapeutic strategies for DR $[32,33]$. In addition, hyperglycemia itself leads to intracellular accumulation of glyceraldehyde- 3 phosphate. Glyceraldehyde-3 phosphate further induces the production of ROS and leads to subsequent accumulation of poly(ADPribose) polymerase-1 (PARP), which triggers the activation of the PKC signaling pathway and increases AGE formation [34]. Indeed, activation of PKC signaling pathway and overproduction of ROS by the mitochondria electron transport chain are the key factors in the pathogenesis of DR $[19,23]$.
Oxidative stress causes the impairment of mitochondrial function by damaging the inner membrane of mitochondria, which leads to imbalance in the electron transport chain and consequently leads to further overproduction of superoxide, ONOO and hydroxyl radicals $[35,36]$. Studies have shown that ROS also impairs mitochondrial function and transport machinery by damaging the mitochondrial DNA and increases apoptosis of retinal capillary cells during DR [37-39]. Increased ROS levels also trigger the release of cytochrome c, which in turn damages the mitochondrial membrane potential and initiate apoptosis by the activation of caspase- 9 and caspase-3 [40-43]. Apoptosis in DR is also regulated by the family of Bcl-2 signaling proteins [43-45].

Several signaling pathways are involved in the pathogenesis of DR. Mitogen-activated protein kinase (MAPK) has been shown to play a role in hyperglycemia-induced cell death in DR [46]. In a cell culture model of hyperglycemia and hypoxia, phosphorylation of c-Jun N-terminal kinases (JNK) and p38 MAPK is induced, leading to overproduction of ROS and disruption of tight junctions in ARPE-19 cells [47]. In streptozotocin-induced diabetic mice, activation of cannabinoid-1 receptor contributes to DR by increasing MAPK activation, oxidative stress, and inflammatory signaling [48]. In addition, PARP and nuclear factor-kappa $\mathrm{B}(\mathrm{NF} \kappa \mathrm{B})$ are also downstream effectors of oxidative stress. PARP is a DNA nick-sensor enzyme that is activated by DNA single-strand breaks. It is involved in DNA repair, gene transcription, cell death, and apoptosis and acts as a coactivator in $\mathrm{NF} \kappa \mathrm{B}$ signaling pathway [34, $49,50]$. Increased levels of oxidative and nitrosative stress induce DNA single-strand break and activates PARP. This in turn inhibits the activity of GAPDH by poly(ADPribosylation) and consequently leads to endothelial dysfunction in diabetic conditions [34]. Therefore, inhibition of PARP could decrease the activation of PKC isoforms, hexosamine pathway flux and AGE formation induced by hyperglycemia [34]. As oxidative stress activates PARP, which in turn initiates the activation $\mathrm{NF} \kappa \mathrm{B}$, application of PARP inhibitor could successfully inhibit hyperglycemia-activated $\mathrm{NF} \kappa \mathrm{B}$ signaling pathway in endothelia cell culture [51]. Also, inhibition of PARP or $\mathrm{NF} \kappa \mathrm{B}$ reduces the hyperglycemiainduced cell death in retinal endothelial cells [50]. NF $\kappa B$ also plays a role in apoptosis by triggering the Notch-1/Akt and PI3k/Akt signaling pathways. Increased activation of PARP, cleaved caspase- 3 as well as reduced expression of Notch1 and pAkt have been observed, which consequently leads to apoptosis in hyperglycemic retina [49].

\section{Oxidative Stress and the Pathogenesis of Retinopathy of Prematurity (ROP)}

Similar to DR, retinal ischemia/hypoxia is a feature in ROP, which is a leading cause of blindness in children worldwide. Retinopathy of prematurity (ROP) was first described as a disease of prematurity $[52,53]$ and contributed to $50 \%$ of infant blindness in 1950. The percentage dropped to $4 \%$ in 1965 after identification of supplemental oxygen use as a 
risk factor. Yet, with improved neonatal care and increased survival of very low birth weight preterm infants the incidence of ROP increased in recent years. ROP is now a major cause of childhood blindness in developed and developing countries. Latest estimates from National Eye Institute showed that $1,100-1,500$ infants $(\sim 5 \%$ infants $\leq 1.25 \mathrm{~kg}$ at birth, $<31$ weeks of gestation) develop severe ROP that requires treatment. Despite treatment, 400-600 infants (40\% with ROP, NIH figure) become legally blind. The resulting long-term disability and severely affected quality of life in ROP patients pose intense burden on the healthcare system worldwide as the population ages, making ROP a major public health issue.

ROP is an ocular disease characterized by vascular abnormalities induced by two phases of pathological changes. The first phase starts with the cessation of retinal vessel growth after premature birth. As premature infants are exposed to high oxygen inside the incubator after birth, a condition of relative hyperoxia happens, leading to downregulation of vascular endothelial growth factor (VEGF) and a subsequent regression of developed retinal vessels. After the cessation of oxygen therapy, infants are returned to normal oxygen tension and a condition of relative hypoxia occurs. In addition, the metabolic demand of the relatively vasculardepleted retina is now higher as the infant grows. Therefore, the increased demand for oxygen poses a hypoxic injury to the retina. The relatively hypoxic condition of the retina triggers the abnormal proliferation of vessels and leads to neovascularization; this is the second phase of ROP progression [54]. Hypoxia-induced oxidative stress therefore, plays a crucial role in the pathogenesis of ROP (Figure 2).

It has been shown that the production of enzymatic and nonenzymatic antioxidants such as SOD, catalase, GPx, vitamin $C$, and vitamin $E$ increases dramatically in the late stage of gestation $[55,56]$. Yet, premature infants are born before term; they are therefore, more susceptible to oxidative stressinduced damage due to the inadequate levels of antioxidants in premature birth and the inability of antioxidant synthesis during the high-oxygen intensive care period [54]. Indeed, the levels of 8-hydroxy 2-deoxyguanosine (8-OHdG), an indicator of oxidative stress, in leukocyte and urine in ROP infants are significant higher than the full-term infants, confirming the presence of oxidative stress in ROP patients [57].

During the two phases of ROP, the retina is subjected to fluctuating oxygen tensions, resulting in retinal hypoxia that triggers the overproduction of ROS, which activates NADPH oxidase and attributes to intravitreal neovascularization by the activation of signaling pathway such as JAK/STAT [58, 59]. On the other hand, inhibition of NADPH oxidase and JAK/STAT signaling pathway reduce the level of cleaved caspase- 3 and hence, apoptosis and neovascularization in $\mathrm{ROP}$ retina $[58,59]$. Increase intake of omega-3-polyunsaturated fatty acids has been shown to reduce the vaso-obliteration and neovascularization by suppressing the TNF- $\alpha$ in ROP retina [60]. Antioxidants such as vitamin E could also decrease the vaso-obliteration

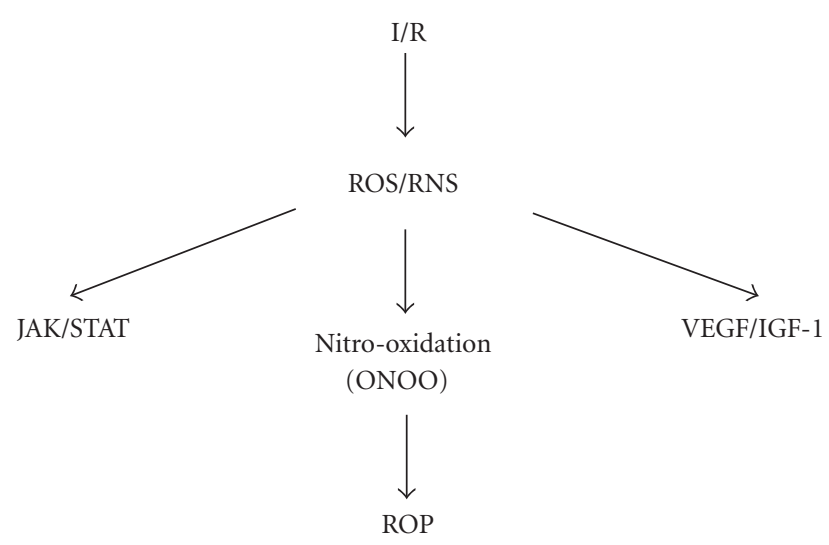

FIGURE 2: Schematic showing the relationship of $\mathrm{I} / \mathrm{R}$, oxidative stress, and ROP. I/R causes overproduction of ROS/RNS. The resulting changes in levels of JAK/STAT, nitro-oxidation, and VEGF/IGF-1 contribute to pathogenesis of ROP.

in ROP retina by its ROS scavenging property [61]. All this evidence points to the importance of oxidative stress in the pathogenesis of ROP.

Nitro-oxidation also contributes to the pathogenesis of ROP. Increased level of NOS, which contributes to the increase of $\mathrm{NO}$ production, is observed in neonatal retina exposed to hypoxia [62]. In an animal model of ROP, increased formation of $\mathrm{ONOO}$ and apoptosis of endothelial cell were seen in association with increased tyrosine nitration of PI3K, cleaved caspase-3, activation of p38 MAPK signaling pathway, and decreased Akt phosphorylation. However, blocking tyrosine nitration of PI3K with epicatechin or $\mathrm{N}$ acetylcysteine reverses the nitro-oxidation-induced pathogenesis [63]. In addition, application of NOS inhibitor or gene deletion of endothelial NOS effectively reduces the severity of ROP in mice, indicating the crucial role of nitrooxidative stress in ROP [64]. Moreover, melatonin administration reduces the production of VEGF and NO as well as promotes cell proliferation, consequently reducing hypoxiaassociated retinopathy [62].

VEGF signaling is a dominant pathway involved in the hypoxia-induced neovascularization and retinopathy in ROP. In the retina, VEGF is primarily secreted by Muller cells and astrocytes. In phase II of ROP, relative hypoxia induces VEGF production and hence promotes pathological vessel proliferation $[65,66]$. IGF-1 is another determining factor in the pathogenesis of ROP and is critical in normal retinal vessel growth. IGF-I is a permissive factor of VEGF activation of Akt survival signaling in the growth of endothelial cells in phase I of ROP [67]. In preterm infants after birth, lower levels of IGF-I reduce Akt activation and endothelial cell survival, which makes the maturing avascular retina becomes hypoxic and leads to accumulation of VEGF in the vitreous [67]. IGF-1 level rises with high levels of VEGF, which triggers neovascularization through the activation of MAPK and Akt signaling pathways in phase II of ROP $[67,68]$. Inhibition of IGF-1 and VEGF are capable to hinder the neovascularization. 


\section{Antioxidants in Ischemic Retinopathies}

DR and ROP are blinding disorders that affect working-age adults and premature infants, causing enormous burden on the society. Yet, the treatment options are limited to laser photocoagulation and anti-VEGF therapy. Pathologically, they both follow a similar pattern as in ischemic retinopathy. As oxidative stress play a pivotal role in the pathogenesis of ischemic retinopathy, agents that can reduce the formation of ROS or increase the antioxidant mechanisms to counteract oxidative stress are beneficial to the situation. Examples of some of these agents are discussed below.

Catalase and SOD are potent ROS scavengers to block oxidative stress in the retina. SOD catalyzes the conversion of superoxide to oxygen and $\mathrm{H}_{2} \mathrm{O}_{2}$ while catalase prevents the formation of hydroxyl radical resulting from the reaction of $\mathrm{H}_{2} \mathrm{O}_{2}$ and ferrous ions [69]. Gene transfer of plasmids encoding MnSOD or catalase complexed with liposomes reduces apoptosis of retinal vascular cell and prevents retinal degeneration induced by retinal I/R [69]. In addition, pretreatment of intravitreal recombinant adenoassociated virus containing the catalase gene (AAV-CAT) efficiently increases the catalase activity and decreases the levels of $\mathrm{H}_{2} \mathrm{O}_{2}, 8$ OHdG and nitrotyrosine as well as attenuates the I/Rinduced retinal function loss, suggesting the beneficial effects of catalase in ischemia/reperfusion-related eye diseases by reducing oxidative stress [70].

As discussed earlier, nitrosative stress also causes detrimental damages during retinal ischemia. NADPH oxidase catalyzes the formation of superoxide from oxygen and NADPH. In ischemic injury in mice, gene deletion of NADPH oxidase reduces the production of ROS, retinal cell loss and glial cell activation, together with an inhibition in the activation of extracellular-signal-regulated kinase (ERK) and $\mathrm{NF} \kappa \mathrm{B}$ signaling pathways [71]. Moreover, blockade of astrocytic NF $\kappa$ B signaling or administration of NADPH oxidase inhibitors reduces retinal ganglion cell death cocultured with astroglia under hypoxia [72]. Gene deletion of astroglial $\mathrm{NF}-\kappa \mathrm{B}$ also reduced oxidative stress in retinal $\mathrm{I} / \mathrm{R}$ in mice $[72,73]$. Most importantly, inhibition of $\mathrm{NF} \kappa \mathrm{B}$ activation by either gene deletion of NADPH oxidase or application of apocynin reduced the retinopathy in ROP and DR animal models [74].

Edaravone is a free radical scavenger which directly scavenges hydroxyl radical and inhibits lipoxygenase activity [75]. It has been shown that application of edaravone can reduce oxidative stress-induced damage by lowering the levels of malondialdehyde (MDA), raising enzymatic activity of SOD, reducing apoptosis of retinal neurons, and preventing retinal functional deterioration in retinal $I / R$ [76]. This suggests that edaravone protects the eye from $\mathrm{I} / \mathrm{R}$ injury and may carry therapeutic potential in treatment of I/R-induced eye disorders.

Vitamin $\mathrm{E}$ is well known to have potent anti-oxidative properties in ocular disease related to retinal $\mathrm{I} / \mathrm{R}$, including ROP and diabetic retinopathy [77-81]. It is a lipid peroxyl radical scavenger via breaking the lipid peroxidation chain and acts against oxidative stress [77-81]. In an animal model of retinal I/R injury, reduced levels of oxidative stress marker such as MDA together with an inactivation of caspase-3 but increased levels of GSH were noted with vitamin E treatment [77-81]. In experimental DR models, oral intake of antioxidants, including vitamin E, significantly reduced lipid peroxidation and arrested retinal abnormalities in streptozotocin (STZ)-induced diabetic rats [82]. Intake of micronutrients based on the AREDS formula (vitamin E, ascorbic acid, beta-carotene, zinc, and copper) inhibited the increases in nitrotyrosine and inducible NOS and decreases in MnSOD in STZ-induced diabetic rats [83]. In ROP models, vitamin E can reduce the area of vaso-obliteration [61, 84], preserve retinal function [85] and protect the reduction of outer plexiform thickness [86]. Most importantly, vitamin E supplementation decreased the risk of severe retinopathy and blindness in very low birth weight infants [81]. All this evidence points to a beneficial role of vitamin $\mathrm{E}$ in ischemic retinopathy.

Numerous studies have investigated the role of erythropoetin (EPO) in ischemic retinopathy. EPO is a glycoprotein hormone that is involved in the pathogenesis of ROP. EPO level decreases and contributes to vessel obliteration in phase I of ROP; therefore, early administration of exogenous EPO minimizes vessel obliteration and subsequent hypoxiainduced neovascularization and neuron apoptosis by the activation of prosurvival $\mathrm{NF} \kappa \mathrm{B}$ signaling pathway via the EPO receptor [87]. However, the level of EPO mRNA increases during the neovascularization phase when late EPO application promotes pathologic neovascularization [87]. In addition, increased level of VEGF leads to vasoobliteration by suppression EPO expression through the Janus kinase/STAT signaling pathway. Application of exogenous EPO inhibits the activation of STAT3 and hence, reduces the area of vaso-obliteration and consequently neovascularization in phase II of ROP [88]. Furthermore, administration of EPO siRNA suppresses neovascularization during phase II of ROP. Therefore, the time of exogenous treatment/inhibition is very critical to the prevention of ROP pathogenesis [89]. Similarly, EPO also exerts its antioxidative and protective effects in DR. Low-dose EPO inhibits oxidative stress and nitro-oxidative stress in experimental diabetic retina by reducing Ang-2 expression and pericyte loss as well as restoring pAkt and heat shock protein-27 levels [90]. In addition, EPO therapy decreases the formation of acellular capillaries and the loss of pericytes, which prevents the early vascular changes in DR [90]. EPO treatment also decreases VEGF levels and protects microvascular and neuronal damage in the experimental diabetic retina [91].

Lutein is a member of xanthophyll family of carotenoids and is contained in dark green leafy vegetables, such as spinach and kale [92-94]. It is a powerful anti-oxidant due to its unique chemical structure. It is characterized by having a hydroxyl group attached to each end of the molecule, making it more hydrophilic, and therefore, lutein reacts more strongly with singlet oxygen than other carotenoids [95, 96]. Increasing evidence has shown that lutein is neuroprotective in retinal $\mathrm{I} / \mathrm{R}$ injury. We show that lutein prevents the increase of nitrotyrosine and PAR and hence, apoptosis and cell loss in inner retinal neuron in an animal model of retinal 
$\mathrm{I} / \mathrm{R}$ [16]. We further show that lutein can directly protect RGC from $\mathrm{H}_{2} \mathrm{O}_{2}$-induced oxidative stress and cobalt chloride $\left(\mathrm{CoCl}_{2}\right)$-induced hypoxia in vitro [97]. In retina of diabetic mice, lutein reduces the production of ROS, inactivates the $\mathrm{NF} \kappa \mathrm{B}$ signaling pathway, and decreases the levels of oxidative markers, thereby preserving the retinal function [98]. Lutein also prevents ERK activation, synaptophysin reduction, BDNF depletion, and consequently neuronal loss in the diabetic retina [99]. However, supplementation of anti-oxidants, including lutein, is ineffective in preventing ROP in preterm infants $[100,101]$. Yet, the protective effects of lutein in ROP are still debatable due to the complicated pathogenesis of ROP.

\section{Future of Antioxidant Treatment in Ischemic Retinopathy}

DR and ROP are two examples of ischemic retinopathy. As mentioned earlier, DR and ROP are blinding disorders that affect working-age adults and premature infants, respectively. The subsequent long-term disability and poor quality of life pose enormous burden on the society. Much research has been conducted, but with no satisfactory outcome. As discussed by Kowluru and Zhong [18], there are serious challenges although promising results are observed in experimental DR models. Firstly, rodents only display the early retinopathy stages but not the later changes seen in patients with diabetic retinopathy. Secondly, DR is a complex diabetic complication; identification of the best molecular pathway for targeted therapeutic studies is not easy. Yet, the administration of the AREDS formula containing various antioxidants to diabetic rats has provided a significant step forward for antioxidant treatment for DR [83].

In ROP, lutein, vitamin $\mathrm{E}$ and $\mathrm{EPO}$ have all shown hopeful results in experimental models. Similar to the negative results in antioxidant therapy in DR, lutein is not able to yield beneficial effects in two randomized controlled clinical studies [100, 101]. However, vitamin E and EPO have shown some promising outcomes clinically. An early metaanalysis concluded that there is a $52 \%$ reduction in the incidence of Stage 3+ ROP with vitamin E prophylaxis in extremely low birth weight $(<1000 \mathrm{gm})$ infants [80]. In a later clinical study, it is concluded that vitamin $\mathrm{E}$ is proven to be effective in prophylaxis of ROP development, and vitamin E supplementation relates to lower incidence of ROP [102]. Recently, interventions such as increasing retinal EPO and vitamin E supplements have been suggested [103]. Although well-designed randomized clinical trials are necessary before these interventions can be put into clinical practice, they have provided strong evidence that antioxidant therapy does have a potential role in treatment of ROP.

\section{Conclusion}

Hypoxia-induced oxidative stress leads to irreversible retinal damage in ocular diseases in which retinal ischemia is a feature. Treatments that can decrease the production of ROS or increase the ROS scavenging ability of the system may be beneficial to the situation. The positive outcomes in administration of AREDS antioxidants in DR experimental models as well as EPO and vitamin E supplements in ROP infants have strongly indicated the potential efficacy of antioxidant in treating ischemic retinopathy with hypoxiainduced oxidative stress.

\section{List of Abbreviations}

8-OHdG: 8-hydroxy 2-deoxyguanosine

AGE: $\quad$ Advanced glycation end-product

AMD: $\quad$ Age-related macular degeneration

AR: $\quad$ Aldose reductase

$\mathrm{Cu} / \mathrm{Zn}$ SOD: Copper/zinc superoxide dismutase

DR: Diabetic retinopathy

EPO: $\quad$ Erythropoietin

GPx: $\quad$ Glutathione peroxidase

GSH: $\quad$ Glutathione

$\mathrm{H}_{2} \mathrm{O}_{2}$ : Hydrogen peroxide

I/R: Ischemia/reperfusion

JNK: $\quad$ c-Jun $\mathrm{N}$-terminal kinases

MAPK: $\quad$ Mitogen-activated protein kinase

MDA: $\quad$ Malondialdehyde

MnSOD: Manganese superoxide dismutase

NADPH: Nicotinamide adenine dinucleotide

phosphate oxidase

NFאB: $\quad$ Nuclear factor-kappa B

NO: $\quad$ Nitric oxide

NOS: $\quad$ Nitric oxide synthase

ONOO: Peroxynitrite

PARP: $\quad$ Poly(ADP-ribose) polymerase-1

PKC: $\quad$ Protein kinase $C$

PTP: $\quad$ Permeability transition pores

RAGE: Receptor for advanced glycation

end-products

Reactive nitrogen species

ROP: $\quad$ Retinopathy of prematurity

ROS: $\quad$ Reactive oxygen species

SOD: $\quad$ Superoxide dismutase

VEGF: Vascular endothelial growth factor.

\section{References}

[1] W. Sickel, "Electrical and metabolic manifestations of receptor and higher-order neuron activity in vertebrate retina," Advances in Experimental Medicine and Biology, vol. 24, pp. 101-118, 1972.

[2] M. Valko, H. Morris, and M. T. D. Cronin, "Metals, toxicity and oxidative stress," Current Medicinal Chemistry, vol. 12, no. 10, pp. 1161-1208, 2005.

[3] W. Dröge, "Free radicals in the physiological control of cell function," Physiological Reviews, vol. 82, no. 1, pp. 47-95, 2002.

[4] M. J. Steinbeck, A. U. Khan, and M. J. Karnovsky, "Extracellular production of singlet oxygen by stimulated macrophages quantified using 9,10-diphenylanthracene and perylene in a polystyrene film," Journal of Biological Chemistry, vol. 268, no. 21, pp. 15649-15654, 1993.

[5] L. F. Agnez-Lima, J. T. Melo, A. E. Silva et al., "Menck CF. DNA damage by singlet oxygen and cellular protective mechanisms," Mutation Research. In press. 
[6] S. Cuzzocrea, D. P. Riley, A. P. Caputi, and D. Salvemini, "Antioxidant therapy: a new pharmacological approach in shock, inflammation, and ischemia/reperfusion injury," Pharmacological Reviews, vol. 53, no. 1, pp. 135-159, 2001.

[7] B. Chance, H. Sies, and A. Boveris, "Hydroperoxide metabolism in mammalian organs," Physiological Reviews, vol. 59, no. 3, pp. 527-605, 1979.

[8] N. N. Osborne, R. J. Casson, J. P. M. Wood, G. Chidlow, M. Graham, and J. Melena, "Retinal ischemia: mechanisms of damage and potential therapeutic strategies," Progress in Retinal and Eye Research, vol. 23, no. 1, pp. 91-147, 2004.

[9] K. Erdélyi, E. Bakondi, P. Gergely, C. Szabó, and L. Virág, "Pathophysiologic role of oxidative stress-induced poly(ADPribose) polymerase-1 activation: focus on cell death and transcriptional regulation," Cellular and Molecular Life Sciences, vol. 62, no. 7-8, pp. 751-759, 2005.

[10] M. M. Elahi, Y. X. Kong, and B. M. Matata, "Oxidative stress as a mediator of cardiovascular disease," Oxidative Medicine and Cellular Longevity, vol. 2, no. 5, pp. 259-269, 2009.

[11] T. C. Squier, "Oxidative stress and protein aggregation during biological aging," Experimental Gerontology, vol. 36, no. 9, pp. 1539-1550, 2001.

[12] M. Valko, D. Leibfritz, J. Moncol, M. T. D. Cronin, M. Mazur, and J. Telser, "Free radicals and antioxidants in normal physiological functions and human disease," International Journal of Biochemistry and Cell Biology, vol. 39, no. 1, pp. 44-84, 2007.

[13] M. E. Bodrova, I. V. Brailovskaya, G. I. Efron, A. A. Starkov, and E. N. Mokhova, "Cyclosporin A-sensitive decrease in the transmembrane potential across the inner membrane of liver mitochondria induced by low concentrations of fatty acids and $\mathrm{Ca}^{2+}$," Biochemistry, vol. 68, no. 4, pp. 391-398, 2003.

[14] H. Tsutsui, S. Kinugawa, and S. Matsushima, "Mitochondrial oxidative stress and dysfunction in myocardial remodelling," Cardiovascular Research, vol. 81, no. 3, pp. 449-456, 2009.

[15] T. Greco, J. Shafer, and G. Fiskum, "Sulforaphane inhibits mitochondrial permeability transition and oxidative stress," Free Radical Biology \& Medicine, vol. 51, pp. 2164-2171, 2011.

[16] S. Y. Li, Z. J. Fu, H. Ma et al., "Effect of lutein on retinal neurons and oxidative stress in a model of acute retinal ischemia/reperfusion," Investigative Ophthalmology and Visual Science, vol. 50, no. 2, pp. 836-843, 2009.

[17] H. Shibuki, N. Katai, J. Yodoi, K. Uchida, and N. Yoshimura, "Lipid peroxidation and peroxynitrite in retinal ischemiareperfusion injury," Investigative Ophthalmology and Visual Science, vol. 41, no. 11, pp. 3607-3614, 2000.

[18] R. A. Kowluru and Q. Zhong, "Beyond AREDS: is there a place for antioxidant therapy in the prevention/treatment of eye disease?" Investigative Ophthalmology \& Visual Science, vol. 52, pp. 8665-8671, 2011.

[19] Y. Yang, M. R. Hayden, S. Sowers, S. V. Bagree, and J. R. Sowers, "Retinal redox stress and remodeling in cardiometabolic syndrome and diabetes," Oxidative Medicine and Cellular Longevity, vol. 3, no. 6, pp. 392-403, 2010.

[20] M. D. Davis, "Diabetic retinopathy: a clinical overview," Diabetes Care, vol. 15, no. 12, pp. 1844-1874, 1992.

[21] A. K. H. Cheung, A. C. Y. Lo, K. F. So, S. S. M. Chung, and S. K. Chung, "Gene deletion and pharmacological inhibition of aldose reductase protect against retinal ischemic injury," Experimental Eye Research, vol. 85, no. 5, pp. 608-616, 2007.

[22] M. Brownlee, "Biochemistry and molecular cell biology of diabetic complications," Nature, vol. 414, no. 6865, pp. 813$820,2001$.
[23] M. Brownlee, "The pathobiology of diabetic complications: a unifying mechanism," Diabetes, vol. 54, no. 6, pp. 1615-1625, 2005.

[24] T. Hattori, A. Matsubara, K. Taniguchi, and Y. Ogura, "Aldose reductase inhibitor fidarestat attenuates leukocyte-endothelial interactions in experimental diabetic rat retina in vivo," Current Eye Research, vol. 35, no. 2, pp. 146-154, 2010.

[25] I. G. Obrosova, A. G. Minchenko, R. Vasupuram et al., "Aldose reductase inhibitor fidarestat prevents retinal oxidative stress and vascular endothelial growth factor overexpression in streptozotocin-diabetic rats," Diabetes, vol. 52, no. 3, pp. 864-871, 2003.

[26] N. Kato, S. Yashima, T. Suzuki, Y. Nakayama, and T. Jomori, "Long-term treatment with fidarestat suppresses the development of diabetic retinopathy in STZ-induced diabetic rats," Journal of Diabetes and its Complications, vol. 17, no. 6, pp. 374-379, 2003.

[27] I. G. Obrosova, Y. Maksimchyk, P. Pacher et al., "Evaluation of the aldose reductase inhibitor fidarestat on ischemia-reperfusion injury in rat retina," International Journal of Molecular Medicine, vol. 26, no. 1, pp. 135-142, 2010.

[28] C. D. Agardh, E. Agardh, I. G. Obrosova, and M. L. Smith, "The aldose reductase inhibitor fidarestat suppresses ischemia-reperfusion- induced inflammatory response in Rat Retina," Pharmacology, vol. 84, no. 5, pp. 257-263, 2009.

[29] M. Nitti, A. L. Furfaro, N. Traverso et al., "PKC delta and NADPH oxidase in AGE-induced neuronal death," Neuroscience Letters, vol. 416, no. 3, pp. 261-265, 2007.

[30] M. Nitti, C. D’Abramo, N. Traverso et al., "Central role of $\mathrm{PKC} \delta$ in glycoxidation-dependent apoptosis of human neurons," Free Radical Biology and Medicine, vol. 38, no. 7, pp. 846-856, 2005.

[31] R. Pazdro and J. R. Burgess, "Differential effects of alphatocopherol and $\mathrm{N}$-acetyl-cysteine on advanced glycation end product-induced oxidative damage and neurite degeneration in SH-SY5Y cells," Biochimica et Biophysica Acta, vol. 1822, pp. 550-556, 2012.

[32] S. I. Yamagishi, S. Amano, Y. Inagaki, T. Okamoto, M. Takeuchi, and Z. Makita, "Beraprost sodium, a prostaglandin I2 analogue, protects against advanced glycation end products-induced injury in cultured retinal pericytes," Molecular Medicine, vol. 8, no. 9, pp. 546-550, 2002.

[33] S. I. Yamagishi, "Role of advanced glycation end products (AGEs) and receptor for AGEs (RAGE) in vascular damage in diabetes," Experimental Gerontology, vol. 46, no. 4, pp. 217224, 2011.

[34] P. Pacher and C. Szabó, "Role of poly(ADP-ribose) polymerase-1 activation in the pathogenesis of diabetic complications: endothelial dysfunction, as a common underlying theme," Antioxidants and Redox Signaling, vol. 7, no. 11-12, pp. 1568-1580, 2005.

[35] R. Radi, A. Cassina, R. Hodara, C. Quijano, and L. Castro, "Peroxynitrite reactions and formation in mitochondria," Free Radical Biology and Medicine, vol. 33, no. 11, pp. 14511464, 2002.

[36] R. Radi, A. Cassina, and R. Hodara, "Nitric oxide and peroxynitrite interactions with mitochondria," Biological Chemistry, vol. 383, no. 3-4, pp. 401-409, 2002.

[37] R. A. Kowluru, V. Kowluru, Y. Xiong, and Y. S. Ho, “Overexpression of mitochondrial superoxide dismutase in mice protects the retina from diabetes-induced oxidative stress," Free Radical Biology and Medicine, vol. 41, no. 8, pp. 11911196, 2006 
[38] R. A. Kowluru, L. Atasi, and Y. S. Ho, "Role of mitochondrial superoxide dismutase in the development of diabetic retinopathy," Investigative Ophthalmology and Visual Science, vol. 47, no. 4, pp. 1594-1599, 2006.

[39] Q. Zhong and R. A. Kowluru, "Diabetic retinopathy and damage to mitochondrial structure and transport machinery," Investigative Ophthalmology \& Visual Science, vol. 52, pp. 8739-8746, 2011.

[40] S. Phaneuf and C. Leeuwenburgh, "Cytochrome c release from mitochondria in the aging heart: a possible mechanism for apoptosis with age," American Journal of Physiology, vol. 282, no. 2, pp. R423-R430, 2002.

[41] F. De Giorgi, L. Lartigue, M. K. Bauer et al., "The permeability transition pore signals apoptosis by directing Bax translocation and multimerization," The FASEB Journal, vol. 16, no. 6, pp. 607-609, 2002.

[42] C. D. Anuradha, S. Kanno, and S. Hirano, "Oxidative damage to mitochondria is a preliminary step to caspase-3 activation in fluoride-induced apoptosis in HL-60 cells," Free Radical Biology and Medicine, vol. 31, no. 3, pp. 367-373, 2001.

[43] R. A. Kowluru, "Diabetic retinopathy: mitochondrial dysfunction and retinal capillary cell death," Antioxidants and Redox Signaling, vol. 7, no. 11-12, pp. 1581-1587, 2005.

[44] T. Oshitari, S. Yamamoto, N. Hata, and S. Roy, "Mitochondria- and caspase-dependent cell death pathway involved in neuronal degeneration in diabetic retinopathy," British Journal of Ophthalmology, vol. 92, no. 4, pp. 552-556, 2008.

[45] T. S. Kern, Y. Du, C. M. Miller, D. A. Hatala, and L. A. Levin, "Overexpression of Bcl-2 in vascular endothelium inhibits the microvascular lesions of diabetic retinopathy," American Journal of Pathology, vol. 176, no. 5, pp. 2550-2558, 2010.

[46] P. Geraldes, J. Hiraoka-Yamamoto, M. Matsumoto et al., "Activation of PKC-and SHP-1 by hyperglycemia causes vascular cell apoptosis and diabetic retinopathy," Nature Medicine, vol. 15, no. 11, pp. 1298-1306, 2009.

[47] S. Miranda, A. Gonzalez-Rodriguez, M. Garcia-Ramirez et al., "Beneficial effects of fenofibrate in retinal pigment epithelium by the modulation of stress and survival signaling under diabetic conditions," Journal of Cellular Physiology, vol. 227, pp. 2352-2362, 2012.

[48] A. B. El-Remessy, M. Rajesh, P. Mukhopadhyay et al., "Cannabinoid 1 receptor activation contributes to vascular inflammation and cell death in a mouse model of diabetic retinopathy and a human retinal cell line," Diabetologia, vol. 54, pp. 1567-1578, 2011.

[49] X. Qin, Z. Zhang, H. Xu, and Y. Wu, "Notch signaling protects retina from nuclear factor-kappaB- and poly-ADPribose-polymerase-mediated apoptosis under high-glucose stimulation," Acta Biochimica et Biophysica Sinica, vol. 43, pp. 703-711, 2011.

[50] L. Zheng, C. Szabó, and T. S. Kern, "Poly(ADP-ribose) polymerase is involved in the development of diabetic retinopathy via regulation of nuclear factor- $\kappa \mathrm{B}$," Diabetes, vol. 53 , no. 11, pp. 2960-2967, 2004.

[51] F. Garcia Soriano, L. Virág, P. Jagtap et al., "Diabetic endothelial dysfunction: the role of poly(ADP-ribose) polymerase activation," Nature Medicine, vol. 7, no. 1, pp. 108-113, 2001.

[52] T. L. Terry, "Fibroblastic overgrowth of persistent tunica vasculosa lentis in infants born prematurely: II. Report of casesclinical aspects," Transactions of the American Ophthalmological Society, vol. 40, pp. 262-284, 1942.

[53] T. L. Terry, "Extreme prematurity and fibroblastic overgrowth of persistent vascular sheath behind each crystalline lens. I. Preliminary report," American Journal of Ophthalmology, vol. 25, no. 2, pp. 203-204, 1942.

[54] J. C. Rivera, P. Sapieha, J. S. Joyal et al., "Understanding retinopathy of prematurity: update on pathogenesis," Neonatology, vol. 100, pp. 343-353, 2011.

[55] L. Frank and E. E. Groseclose, "Preparation for birth into an $\mathrm{O}_{2}$-rich environment: the antioxidant enzymes in the developing rabbit lung," Pediatric Research, vol. 18, no. 3, pp. 240244, 1984.

[56] S. Qanungo and M. Mukherjea, "Ontogenic profile of some antioxidants and lipid peroxidation in human placental and fetal tissues," Molecular and Cellular Biochemistry, vol. 215, no. 1-2, pp. 11-19, 2000.

[57] O. Ates, H. H. Alp, I. Caner et al., "Oxidative DNA damage in retinopathy of prematurity," European Journal of Ophthalmology, vol. 19, no. 1, pp. 80-85, 2009.

[58] G. Byfield, S. Budd, and M. Elizabeth Hartnett, "The role of supplemental oxygen and JAK/STAT signaling in intravitreous neovascularization in a ROP rat model," Investigative Ophthalmology and Visual Science, vol. 50, no. 7, pp. 33603365, 2009.

[59] M. Elizabeth Hartnett, "The effects of oxygen stresses on the development of features of severe retinopathy of prematurity: knowledge from the 50/10 OIR model," Documenta Ophthalmologica, vol. 120, no. 1, pp. 25-39, 2010.

[60] K. M. Connor, J. P. Sangiovanni, C. Lofqvist et al., "Increased dietary intake of $\omega$-3-polyunsaturated fatty acids reduces pathological retinal angiogenesis," Nature Medicine, vol. 13, no. 7, pp. 868-873, 2007.

[61] J. S. Penn, L. A. Thum, and M. I. Naash, "Oxygen-induced retinopathy in the rat: vitamins $\mathrm{C}$ and $\mathrm{E}$ as potential therapies," Investigative Ophthalmology and Visual Science, vol. 33, no. 6, pp. 1836-1845, 1992.

[62] C. Kaur, V. Sivakumar, W. S. Foulds, C. D. Luu, and E. A. Ling, "Cellular and vascular changes in the retina of neonatal rats after an acute exposure to hypoxia," Investigative Ophthalmology and Visual Science, vol. 50, no. 11, pp. 5364-5374, 2009.

[63] M. A. Abdelsaid, B. A. Pillai, S. Matragoon, R. Prakash, M. Al-Shabrawey, and A. B. El-Remessy, "Early intervention of tyrosine nitration prevents vaso-obliteration and neovascularization in ischemic retinopathy," Journal of Pharmacology and Experimental Therapeutics, vol. 332, no. 1, pp. 125-134, 2010.

[64] S. E. Brooks, X. Gu, S. Samuel et al., "Reduced severity of oxygen-induced retinopathy in eNOS-deficient mice," Investigative Ophthalmology and Visual Science, vol. 42, no. 1, pp. 222-228, 2001.

[65] W. T. Kim and E. S. Suh, "Retinal protective effects of resveratrol via modulation of nitric oxide synthase on oxygeninduced retinopathy," Korean Journal of Ophthalmology, vol. 24, no. 2, pp. 108-118, 2010.

[66] C. Romagnoli, "Risk factors and growth factors in ROP," Early Human Development, vol. 85, pp. S79-S82, 2009.

[67] A. Hellstrom, C. Perruzzi, M. Ju et al., "Low IGF-I suppresses VEGF-survival signaling in retinal endothelial cells: direct correlation with clinical retinopathy of prematurity," Proceedings of the National Academy of Sciences of the United States of America, vol. 98, no. 10, pp. 5804-5808, 2001.

[68] E. Kermorvant-Duchemin, P. Sapieha, M. Sirinyan et al., "Understanding ischemic retinopathies: emerging concepts from oxygen-induced retinopathy," Documenta Ophthalmologica, vol. 120, no. 1, pp. 51-60, 2010. 
[69] B. Chen, S. Caballero, S. Seo, M. B. Grant, and A. S. Lewin, "Delivery of antioxidant enzyme genes to protect against ischemia/reperfusion-induced injury to retinal microvasculature," Investigative Ophthalmology and Visual Science, vol. 50, no. 12, pp. 5587-5595, 2009.

[70] B. Chen and L. Tang, "Protective effects of catalase on retinal ischemia/reperfusion injury in rats," Experimental Eye Research, vol. 93, pp. 599-606, 2011.

[71] H. Yokota, S. P. Narayanan, W. Zhang et al., "Neuroprotection from retinal ischemia/reperfusion injury by NOX2 NADPH oxidase deletion," Investigative Ophthalmology \& Visual Science, vol. 52, pp. 8123-8131, 2011.

[72] D. J. Barakat, G. Dvoriantchikova, D. Ivanov, and V. I. Shestopalov, "Astroglial NF-kappaB mediates oxidative stress by regulation of NADPH oxidase in a model of retinal ischemia reperfusion injury," Journal of Neurochemistry, vol. 120, pp. 586-597, 2012.

[73] G. Dvoriantchikova, D. Barakat, R. Brambilla et al., "Inactivation of astroglial NF- $\kappa$ B promotes survival of retinal neurons following ischemic injury," European Journal of Neuroscience, vol. 30, no. 2, pp. 175-185, 2009.

[74] A. Tawfik, T. Sanders, K. Kahook, S. Akeel, A. Elmarakby, and M. Al-Shabrawey, "Suppression of retinal peroxisome proliferator-activated receptor $\gamma$ in experimental diabetes and oxygen-induced retinopathy: role of NADPH oxidase," Investigative Ophthalmology and Visual Science, vol. 50, no. 2, pp. 878-884, 2009.

[75] T. Kuzuya, S. Hoshida, Y. Kim et al., "Free radical generation coupled with arachidonate lipoxygenase reaction relates to reoxygenation induced myocardial cell injury," Cardiovascular Research, vol. 27, no. 6, pp. 1056-1060, 1993.

[76] Y. Song, Y. Y. Gong, Z. G. Xie, C. H. Li, Q. Gu, and X. W. Wu, "Edaravone (MCI-186), a free radical scavenger, attenuates retinal ischemia/reperfusion injury in rats," Acta Pharmacologica Sinica, vol. 29, no. 7, pp. 823-828, 2008.

[77] R. Pazdro and J. R. Burgess, "The role of vitamin E and oxidative stress in diabetes complications," Mechanisms of Ageing and Development, vol. 131, no. 4, pp. 276-286, 2010.

[78] O. Aydemir, S. Çelebi, T. Yilmaz, H. Yekeler, and A. Ş. Kükner, "Protective effects of vitamin E forms (alpha-tocopherol, gamma-tocopherol and d-alpha-tocopherol polyethylene glycol 1000 succinate) on retinal edema during ischemiareperfusion injury in the guinea pig retina," International Ophthalmology, vol. 25, no. 5-6, pp. 283-289, 2005.

[79] N. Dilsiz, A. Sahaboglu, M. Z. Yildiz, and A. Reichenbach, "Protective effects of various antioxidants during ischemiareperfusion in the rat retina," Graefe's Archive for Clinical and Experimental Ophthalmology, vol. 244, no. 5, pp. 627-633, 2006.

[80] T. N. K. Raju, P. Langenberg, V. Bhutani, and G. E. Quinn, "Vitamin E prophylaxis to reduce retinopathy of prematurity: a reappraisal of published trials," Journal of Pediatrics, vol. 131, no. 6, pp. 844-850, 1997.

[81] L. P. Brion, E. F. Bell, and T. S. Raghuveer, "Vitamin E supplementation for prevention of morbidity and mortality in preterm infants," Cochrane Database of Systematic Reviews, no. 3, Article ID CD003665, 2003.

[82] F. Yülek, M. Or, C. Özoğul et al., "Effects of stobadine and vitamin $\mathrm{E}$ in diabetes-induced retinal abnormalities: involvement of oxidative stress," Archives of Medical Research, vol. 38, no. 5, pp. 503-511, 2007.

[83] R. A. Kowluru, M. Kanwar, P. S. Chan, and J. P. Zhang, "Inhibition of retinopathy and retinal metabolic abnormalities in diabetic rats with AREDS-based micronutrients," Archives of Ophthalmology, vol. 126, no. 9, pp. 1266-1272, 2008.

[84] J. S. Penn, "Oxygen-induced retinopathy in the rat: possible contribution of peroxidation reactions," Documenta Ophthalmologica, vol. 74, no. 3, pp. 179-186, 1990.

[85] F. Block and M. Schwarz, "The b-wave of the electroretinogram as an index of retinal ischemia," General Pharmacology, vol. 30, no. 3, pp. 281-287, 1998.

[86] A. L. Dorfman, O. Dembinska, S. Chemtob, and P. Lachapelle, "Structural and functional consequences of trolox C treatment in the rat model of postnatal hyperoxia," Investigative Ophthalmology and Visual Science, vol. 47, no. 3, pp. 1101-1108, 2006.

[87] J. Chen, K. M. Connor, C. M. Aderman, and L. E. H. Smith, "Erythropoietin deficiency decreases vascular stability in mice," Journal of Clinical Investigation, vol. 118, no. 2, pp. 526-533, 2008.

[88] H. Wang, G. Byfield, Y. Jiang, G. W. Smith, M. McCloskey, and M. E. Hartnett, "VEGF-mediated STAT3 activation inhibits retinal vascularization by down-regulating local erythropoietin expression," The American Journal of Pathology, vol. 180, pp. 1243-1253, 2012.

[89] C. Romagnoli, M. G. Tesfagabir, C. Giannantonio, and P. Papacci, "Erythropoietin and retinopathy of prematurity," Early Human Development, vol. 87, supplement 1, pp. S39-S42, 2011.

[90] Q. Wang, F. Pfister, A. Dorn-Beineke et al., "Low-dose erythropoietin inhibits oxidative stress and early vascular changes in the experimental diabetic retina," Diabetologia, vol. 53, no. 6, pp. 1227-1238, 2010.

[91] Q. Wang, S. Gorbey, F. Pfister et al., "Long-term treatment with suberythropoietic epo is vaso- and neuroprotective in experimental diabetic retinopathy," Cellular Physiology and Biochemistry, vol. 27, no. 6, pp. 769-782, 2011.

[92] O. Sommerburg, J. E. E. Keunen, A. C. Bird, and F. J. G. M. Van Kuijk, "Fruits and vegetables that are sources for lutein and zeaxanthin: the macular pigment in human eyes," British Journal of Ophthalmology, vol. 82, no. 8, pp. 907-910, 1998.

[93] N. I. Krinsky, J. T. Landrum, and R. A. Bone, "Biologic mechanisms of the protective role of lutein and zeaxanthin in the eye," Annual Review of Nutrition, vol. 23, pp. 171-201, 2003.

[94] A. R. Mangels, J. M. Holden, G. R. Beecher, M. R. Forman, and E. Lanza, "Carotenoid content of fruits and vegetables: an evaluation of analytic data," Journal of the American Dietetic Association, vol. 93, pp. 284-296, 1993.

[95] F. Ojima, H. Sakamoto, Y. Ishiguro, and J. Terao, "Consumption of carotenoids in photosensitized oxidation of human plasma and plasma low-density lipoprotein," Free Radical Biology and Medicine, vol. 15, no. 4, pp. 377-384, 1993.

[96] J. D. Ribaya-Mercado and J. B. Blumberg, "Lutein and zeaxanthin and their potential roles in disease prevention," Journal of the American College of Nutrition, vol. 23, pp. 567S587S, 2004.

[97] S. Y. Li and A. C. Y. Lo, "Lutein protects RGC-5 cells against hypoxia and oxidative stress," International Journal of Molecular Sciences, vol. 11, no. 5, pp. 2109-2117, 2010.

[98] M. Muriach, F. Bosch-Morell, G. Alexander et al., "Lutein effect on retina and hippocampus of diabetic mice," Free Radical Biology and Medicine, vol. 41, no. 6, pp. 979-984, 2006.

[99] M. Sasaki, Y. Ozawa, T. Kurihara et al., "Neurodegenerative influence of oxidative stress in the retina of a murine model of diabetes," Diabetologia, vol. 53, no. 5, pp. 971-979, 2010.

[100] C. Dani, I. Lori, F. Favelli et al., "Lutein and zeaxanthin supplementation in preterm infants to prevent retinopathy of 
prematurity: a randomized controlled study," Journal of Maternal-Fetal and Neonatal Medicine, vol. 25, pp. 523-527, 2012.

[101] C. Romagnoli, C. Giannantonio, F. Cota et al., "A prospective, randomized, double blind study comparing lutein to placebo for reducing occurrence and severity of retinopathy of prematurity," Journal of Maternal-Fetal and Neonatal Medicine, vol. 24, supplement 1, pp. 147-150, 2011.

[102] P. M. Liu, P. C. Fang, C. B. Huang et al., "Risk factors of retinopathy of prematurity in premature infants weighing less than 1600 g," American Journal of Perinatology, vol. 22, no. 2, pp. 115-120, 2005.

[103] T. S. Raghuveer and B. T. Bloom, "A paradigm shift in the prevention of retinopathy of prematurity," Neonatology, vol. 100, no. 2, pp. 116-129, 2011. 


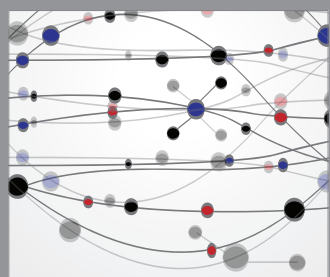

The Scientific World Journal
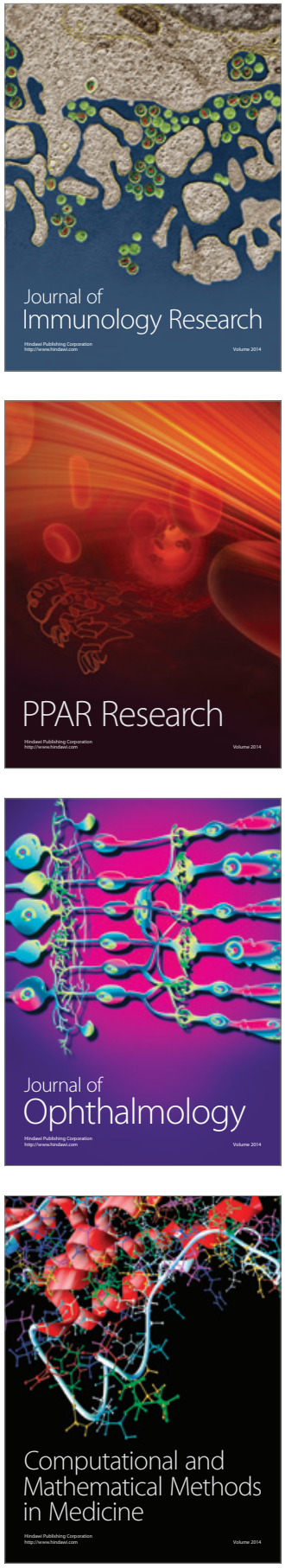

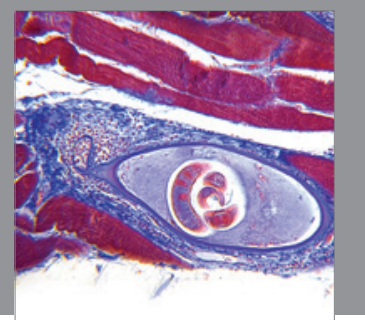

Gastroenterology

Research and Practice
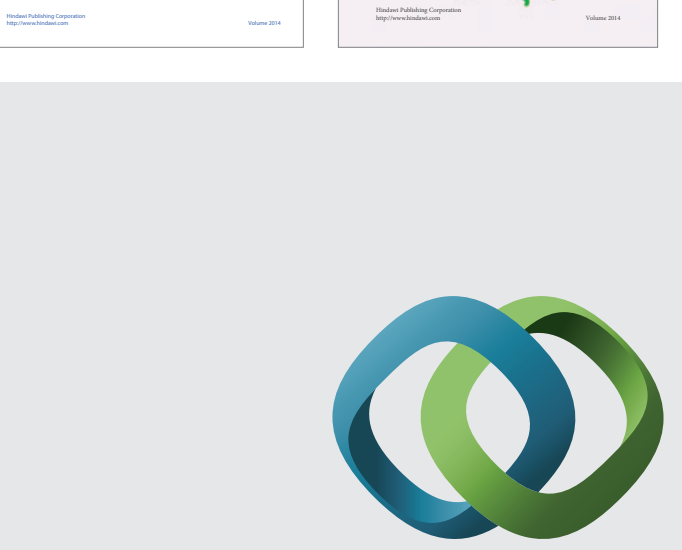

\section{Hindawi}

Submit your manuscripts at

http://www.hindawi.com
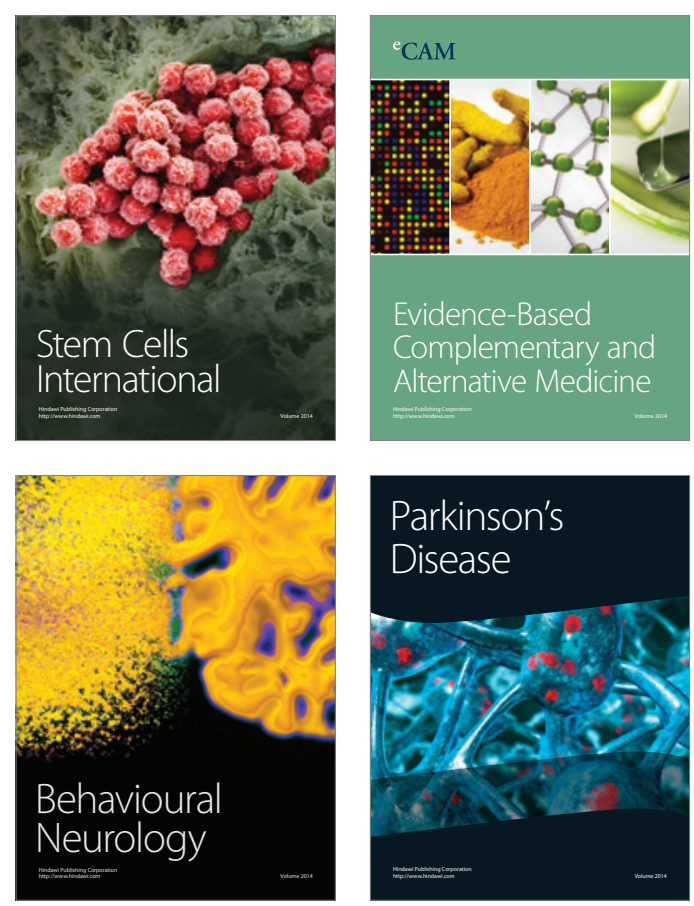

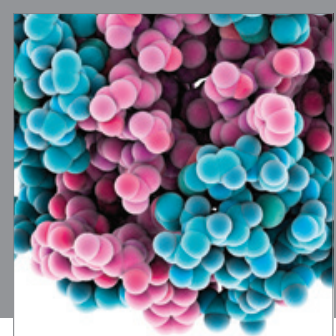

Journal of
Diabetes Research

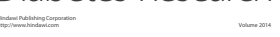

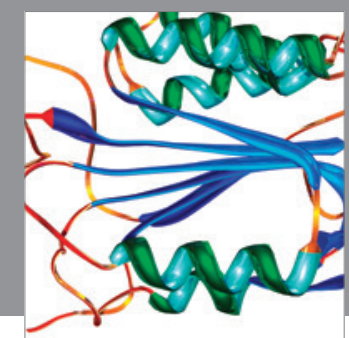

Disease Markers
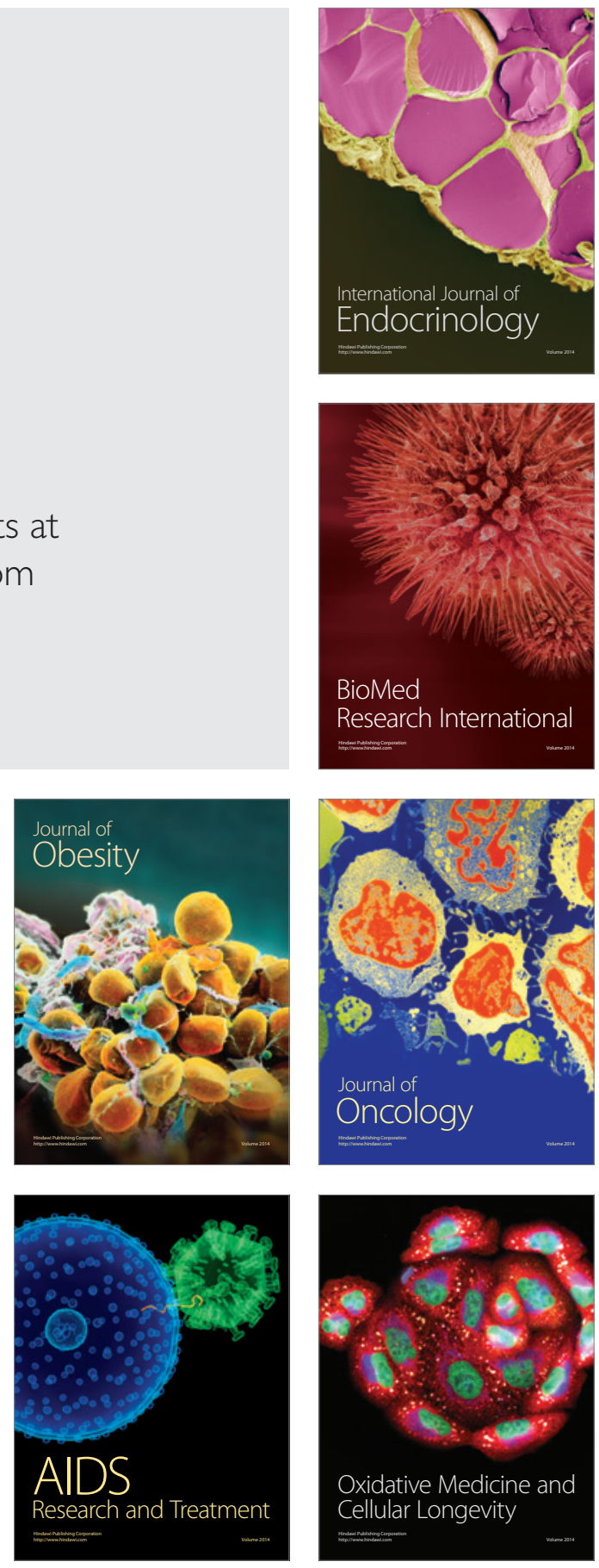